Trauma Berufskrankh 2006 8 [Suppl 3]:S258-S263 DOI 10.1007/s10039-005-1067-x

Online publiziert: 21. Dezember 2005

๑) Springer Medizin Verlag 2005
C. Voigt $\cdot$ H. Lill

Klinik für Unfall- und Wiederherstellungschirurgie, Friederikenstift, Hannover

\title{
Abgrenzungsprobleme bei Schulterluxationen
}

\section{Klassifikation}

Die im klinischen Alltag verbreitetste Klassifikation der Schulterinstabilitäten ist die Gerber-Einteilung [11]. Sie umfasst die dynamischen Instabilitäten (Gerber-NyffelerKlasse B [13]) und differenziert nach Richtung, Hyperlaxität und Willkürlichkeit.

2002 wurde sie von Gerber u. Nyffeler [13] um die Gruppen der statischen Instabilitäten (Gerber-Nyfeller-Klasse A) und willkürlichen Luxationen (Gerber-Nyfeller-Klasse C) erweitert (- Tabelle 1). Als statische Instabilitäten gelten eine fixe superiore, inferiore, anteriore oder posteriore Dezentrierung des Humeruskopfes. Ursächlich können Rotatorenmanschettenläsionen, degenerative oder traumatische Veränderungen, anatomische Glenoidvarianten, Nervenverletzungen oder Längenunterschiede nach prothetischer Versorgung sein $[13,34]$.

\section{Wichtige Definitionen}

- Schulterluxation

Sie ist als eine pathologisch vermehrte

Translation mit komplettem Kontaktverlust der Gelenkflächen definiert.

- Schulterinstabilität

Es liegt eine Unfähigkeit, den Humeruskopf im Glenoid zu zentrieren, vor. Es werden uni- (1 Richtung) und multidirektionale (mindestens 2 Richtungen) Instabilitäten unterschieden.

\section{• Hyperlaxität}

Es handelt sich um eine gesteigerte physiologische Translation. Sie kann einen Risikofaktor für die Entstehung einer Instabilität darstellen oder mit ihr kombiniert sein. Die Hyperlaxität selbst besitzt primär keinen Krankheitswert [34].
Die dynamischen Instabilitäten sind durch ein subjektives Instabilitätsgefühl und einen momentanen reversiblen Verlust der Gelenkkongruenz gekennzeichnet. Sie werden immer durch ein Trauma initialisiert. Dabei können ein Makrotrauma oder repetitive Mikrotraumen vorliegen [13].

95\% die Schulterluxationen sind unidirektional nach anterior-inferior gerichtet. 30\% der unidirektionalen Instabilitäten weisen zusätzlich eine multidirektionale Hyperlaxität auf. In 2-4\% liegt eine hintere Luxation, in 3-5\% eine multidirektionale Instabilität mit Hyperlaxität vor [12, 17].

\section{Diagnostik}

\section{Klinische Untersuchung}

Entscheidende Hinwiese ergeben sich bereits aus der Anamnese. Es gilt

- Ursache (traumatisch-habituell),

- Häufigkeit,

- Richtung (uni-/multidirektional),

- Willkürlichkeit,

- Ausmaß (Sub-/Luxation) und

- Repositionsverhalten (Eigen-/Fremdreposition)

zu erfassen.

Die klinische Untersuchung erfolgt immer im Seitenvergleich und beginnt mit der Beurteilung der Schulterlaxität.

Schulterlaxität. Zunächst werden schulterunspezifische Hyperlaxitätsparameter überprüft. Hinweisend sind die Überstreckbarkeit der Finger- und Ellbogengelenke sowie ein Daumen-Unterarm-Abstand $<6 \mathrm{~cm}[3,34]$. Der Schubladentest evaluiert die anteriore und posteriore Translation, die nach Hawkins u. Bokor [18] graduiert wird (• Tabelle 2). Die inferiore Translation wird im Sulcuszeichen (Neutralposition) erfasst und entspre-

Tabelle 1

Klassifikation der Schulterinstabilität nach Gerber u. Nyffeler [13]

Klasse A - Statische Instabilität

A1 Superior

A2 Anterior

A3 Posterior

A4 Inferior

Klasse B - Dynamische Instabilität

B1 Chronisch verhakte Luxation

B2 Unidirektionale Instabilität ohne Hyperlaxität

B3 Unidirektionale Instabilität mit Hyperlaxität

B4 Multidirektionale Instabilität ohne Hyperlaxität

B5 Multidirektionale Instabilität mit Hyperlaxität

B6 Willkürliche Instabilität

Klasse C - Willkürliche Luxation

Tabelle 2

Einteilung des Translationsgrads nach Hawkins u. Bokor [18]

Grad 0 Keine/geringe Translation

Grad 1 Translation bis an den Glenoidrand

Grad 2 Translation bis zur Hälfte des Kopfdurchmessers auf den Glenoidrand mit Spontanreposition

Grad 3 Translation über den Glenoidrand ohne Spontanreposition 


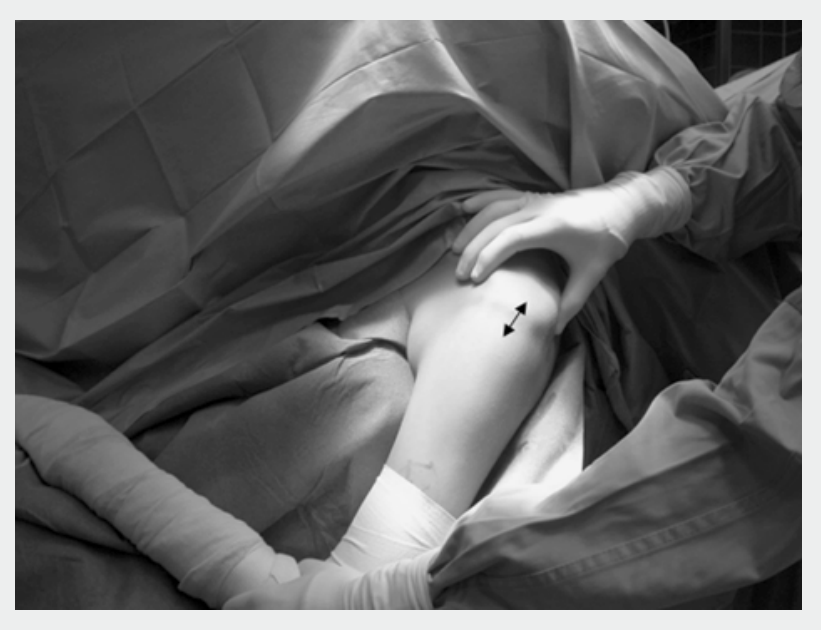

Abb. $1<$ Sulcuszeichen

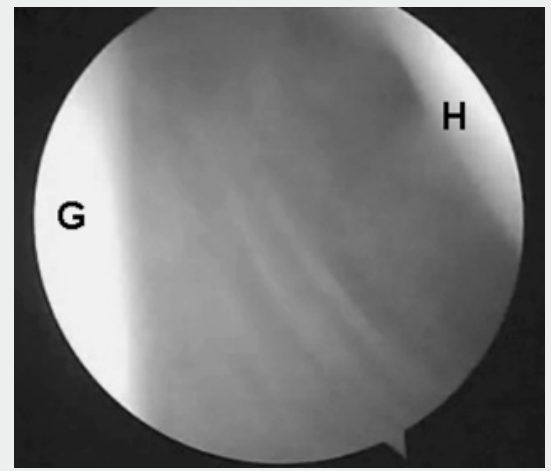

Abb. $2 \triangle$ Drive-through-Phänomen mit weiter Gelenkkapsel bei Hyperlaxität; arthroskopisches Bild, G Glenoid, $H$ Humeruskopf chend seiner Ausdehnung $(0-2 \mathrm{~cm})$ nach Altcheck et al. [1] klassifiziert (• Abb. 1). Ein positives Sulcuszeichen in Außenrotation deutet auf eine Insuffizienz des Rotatorenintervalls, in Innenrotation auf eine Instabilität der dorsalen Kapselstrukturen hin. Eine Hyperlaxität der inferioren Kapsel manifestiert sich in einem positiven $\mathrm{Ga}$ gey-Test (bei fixierter Skapula passive Abduktion $>105^{\circ}$ ) [9].

Schulterinstabilität. Es folgt die Untersuchung der Schulterinstabilität. Die vordere Instabilität wird durch den vorderen Apprehension-Test (Abduktion, Außenrotation und dorsaler Druck auf den Humeruskopf) geprüft. Dieser sollte in 6o, 90 und $120^{\circ}$ Abduktion erfolgen, um das Ausmaß der Kapsel-Labrum-Schädigung zu erfassen. Dabei werden in $60^{\circ}$-Stellung das mittlere, in 90 und $120^{\circ}$ das inferiore glenohumerale Band getestet. Die posteriore Instabilität wird mit dem Jerk-Test $\left(90^{\circ}\right.$ Abduktion, zunehmende Adduktion und Innenrotation unter axialem Druck) beurteilt [18].

Intraartikuläre Verletzungen. Ihr Schweregrad bei einer traumatischen Luxation hängt vom Ausmaß der Laxität ab. Dabei kann der Labrum-Ligament-Komplex am glenoidalen Ansatz (Bankart-Läsion: Labrumabriss, Perthes-Läsion: Riss des medialen Kapselursprungs ohne Labrumläsion), im Verlauf der Kapsel oder am humeralen Ansatz (HAGL-Läsion, HAGL: humerale Avulsion der glenohumeralen Ligamente) geschädigt werden. Zusätzlich wird von einer plastischen Deformierung der Kapsel und glenohumeralen Ligamen- te ausgegangen. Bei spontanem Verlauf kann es nach Bankart-Läsion zu einer medialen Fehlverheilung des Labrum-KapselKomplexes (ALPSA-Läsion, ALPSA: „anterior labroligamentous periosteal sleeve avulsion") kommen [25].

Bei über 30-jährigen Patienten wird nach Erstluxation in bis zu 100\% der Fälle eine Kapsel-Labrum-Läsion beobachtet. 30-80\% der > 40-Jährigen erfahren eine begleitende Rotatorenmanschettenruptur unterschiedlichen Ausmaßes [21, 28], wobei zu berücksichtigen ist, dass die dynamischen Stabilisatoren (Rotatorenmanschette) ab dem 4o. Lebensjahr zunehmend an Bedeutung gewinnen.

Knöcherne Läsionen. Sie werden am Glenoid und korrespondierend am Humeruskopf beobachtet. Bei der vorderen Luxation führt der anterior-inferiore Pfannenrand zu einer Impressionsfraktur am posterolateralen Humeruskopf (Hill-SachsLäsion); entsprechend führt eine dorsale Luxation zu einer Impression am anteromedialen Humeruskopf (Reversed-HillSachs- oder MacLaughlin-Läsion). Abscherfrakturen am anterior-inferioren Pfannenrand werden als Bankart-Frakturen bezeichnet. Diese Defekte sind umso ausgeprägter, je weniger lax das betroffene Schultergelenk konfiguriert ist. So zeigen sich bei hyperlaxen Gelenken keine oder nur wenig ausgeprägte ossäre Läsionen.

Unidirektionale Instabilität. Charakteristisch für die B1-Instabilität nach GerberNyffeler sind ein stattgehabtes Makrotrauma (Motorradunfall, epileptischer Anfall) und eine ausgedehnte Humeruskopfim- pressionsfraktur. Rotatorenmanschettenläsionen oder große Glenoiddefekte sind selten [13].

Die unidirektionalen Instabilitäten ohne Hyperlaxität (B2) sind mit 6o\% aller Instabilitäten am häufigsten. Typisch sind ein stattgehabtes adäquates Trauma mit Fremdreposition, ein positiver Apprehension-Test bei negativem Schubladentest und Sulcuszeichen. Ein großer Hill-SachsDefekt ist mit einer kleinen Läsion des anterior-inferioren Labrumkomplexes assoziiert und umgekehrt [13]. Posteriore Bankart-Läsionen sind weniger ausgedehnt als anteriore; kommen jedoch häufiger vor als früher angenommen [8].

Die unidirektionale Instabilität mit Hyperlaxität $\left(\mathrm{B}_{3}\right)$ liegt in etwa 30\% aller Instabilitäten vor [13]. Durch ein geringes Trauma ausgelöst sind ein positiver anteriorer oder posteriorer Apprehension-Test mit positivem Schubladentest und Sulcuszeichen, eine vermehrte Außenrotation sowie eine hyperlaxe kontralaterale Schulter charakteristisch. Die Kapsel-Labrumund knöchernen Läsionen sind bei großem Kapselvolumen klein [13, 34].

Multidirektionale Instabilität. Bei Patienten mit der sehr seltenen multidirektionalen Instabilität ohne Hyperlaxität (B4) liegen meist 2 unabhängige Unfallereignisse zugrunde. Sie weisen einen positiven vorderen und hinteren Apprehension-Test auf, die Außenrotation liegt nicht $>70^{\circ}$, die Hyperlaxitätszeichen sind negativ. Läsionen der anterioren und posterioren Instabilität werden beobachtet $[13,34]$.

Die multidirektionalen Instabilitäten mit Hyperlaxität (B5) gilt es von der uni- 


\section{Schultergelenkverletzung}
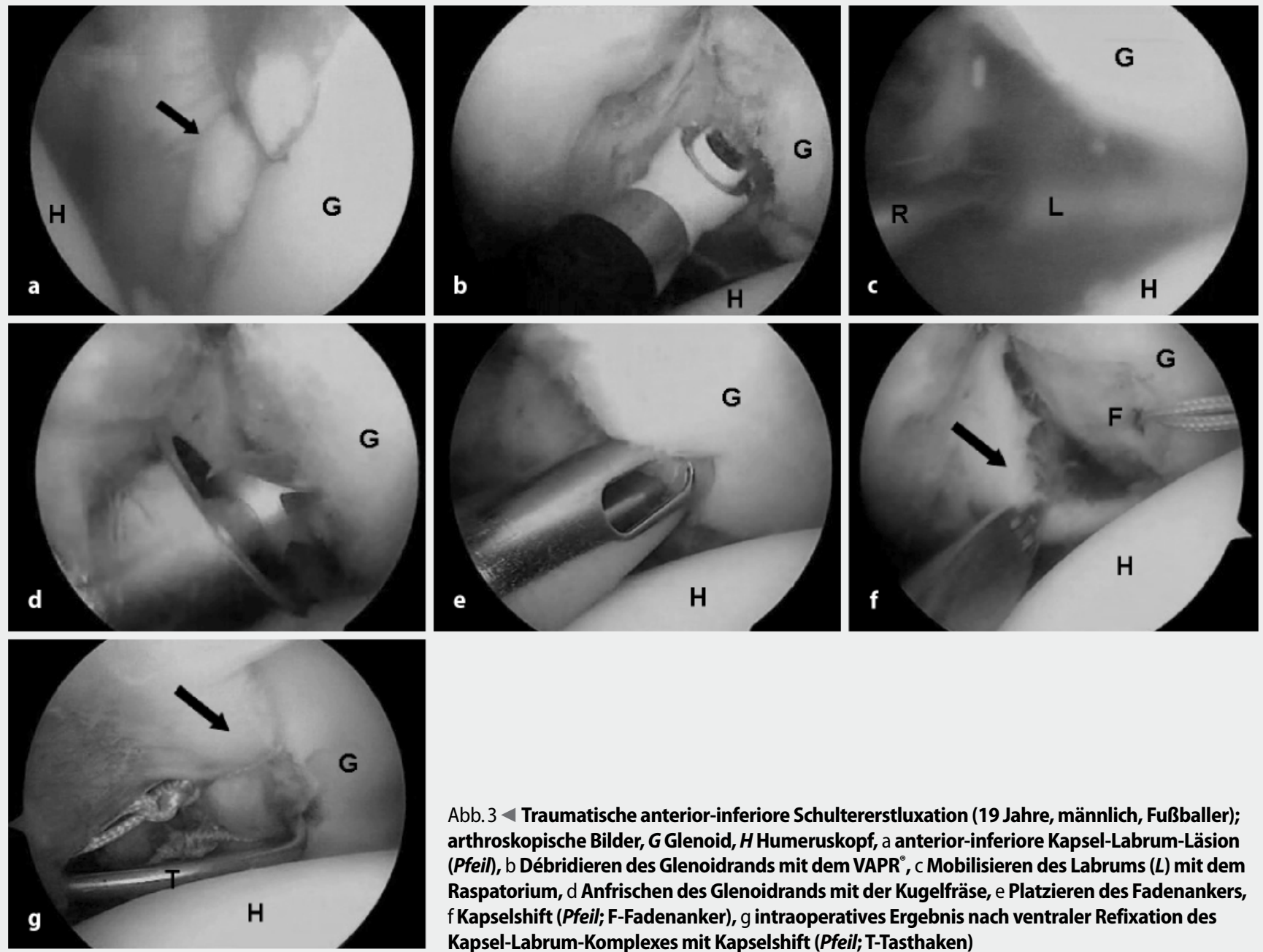

Abb. $3<$ Traumatische anterior-inferiore Schultererstluxation (19 Jahre, männlich, Fußballer); arthroskopische Bilder, $\boldsymbol{G}$ Glenoid, $\boldsymbol{H}$ Humeruskopf, a anterior-inferiore Kapsel-Labrum-Läsion (Pfeil), b Débridieren des Glenoidrands mit dem VAPR ${ }^{\circ}$, c Mobilisieren des Labrums $(L)$ mit dem Raspatorium, $\mathrm{d}$ Anfrischen des Glenoidrands mit der Kugelfräse, e Platzieren des Fadenankers, f Kapselshift (Pfeil; F-Fadenanker), g intraoperatives Ergebnis nach ventraler Refixation des Kapsel-Labrum-Komplexes mit Kapselshift (Pfeil; T-Tasthaken) direktionalen Instabilität mit Hyperlaxität abzugrenzen. Die typischen Patienten sind Frauen mit repetitiven Mikrotraumen in der Jugend (Überkopfsport). Häufig sind beide Schultergelenke betroffen. Bei der klinischen Untersuchung finden sich eine generalisierte Hyperlaxität sowie ein vorderes und hinteres ApprehensionZeichen. Innen- und Außenrotation sind vermehrt. Auffällig sind ein generalisiertes großes Kapselvolumen mit bei der arthoskopischen Untersuchung typischem „Drive-through-Phänomen“ (• Abb. 2), ein weites Rotatorenintervall sowie keine oder nur minimale intraartikuläre Verletzungen [13].

Eine willkürliche Instabilität (B6) liegt bei Patienten vor, die ihre Schulter nach Sub-/Luxation selbst reponieren können. Sie ist in der Regel schmerzfrei und wird bilateral beobachtet $[13,34]$.

\section{Bildgebende Diagnostik}

Röntgen. Grundlage ist die Röntgendiagnostik. Die True-a.-p.-Aufnahme stellt neben der Dislokation den vorderen und hinteren Pfannenrand dar. Knöcherne Begleitverletzungen, wie eine Bankart- oder Tuberculum-majus-Fraktur, können so diagnostiziert werden. Die Y-Aufnahme erlaubt die Differenzierung zwischen ventraler und dorsaler Richtung. Die axiale Darstellung ist bei der akuten Luxation häufig nicht möglich. Eine Alternative zur Beurteilung des Pfannenrandes stellt die Velpeau-Aufnahme dar. Die Hill-Sachs-Läsion lässt sich am besten in der StrykerNotch-Aufnahme, einer modifizierten axialen Aufnahme, verifizieren [25].

\section{Zusätzliche Computertomographie (CT).} Sie wird beim Verdacht auf eine chronisch verhakte Luxation sowie bei unklarem Befund am Glenoid empfohlen und ermög- licht die therapierelevante Größenbestimmung knöcherner Defekte.

Magnetresonanztomographie (MRT). Die MRT erfasst begleitende Labrum-Kapsel-, SLAP- („superior labrum anterior to posterior") und Rotatorenmanschettenläsionen. Die MRT ist nach Schultererstluxation bei älteren Patienten (>40 Jahre) obligat, um die in bis zu 63\% auftretenden begleitenden Rotatorenmanschettenrupturen $\mathrm{zu}$ diagnostizieren [15, 21]. Generell hat die Arthro-MRT gegenüber der Nativ-MRT und der Arthro-CT die höchste Spezifität und Sensitivität [26]. Innerhalb der ersten 2 Wochen nach Trauma genügt durch den Kontrasteffekt des Hämarthros ein Nativ-MRT [25].

Die Indikation zur MRT bei Patienten $<30$ Jahren besteht nur, wenn nach Erstluxation nicht sicher zwischen uni- und multidirektionaler Instabilität mit beglei- 
tender Hyperlaxität unterschieden werden kann [18].

\section{Das Therapieregime beeinflussen- de Prognosefaktoren}

\section{Patientenalter}

Die Reluxationsrate nach konservativer Therapie traumatischer Schultererstluxationen verhält sich umgekehrt proportional zum Patientenalter - je jünger der $\mathrm{Pa}$ tient, desto höher die Reluxationsrate. Die Literatur gibt hier die "Schnittstelle“ von 30 Jahren vor. Lill et al. [27] und Hovelius et al. [21] beschrieben Reluxationsraten von $60-86 \%$ bei <30-jährigen und $12-21 \%$ bei $>30$-jährigen Patienten.

\section{Aktivitätsgrad}

Wheeler et al. [39] sahen Reluxationsraten von 94\% bei Sportlern (Überkopf-, Wurfund Kontaktsportarten) nach konservativer Therapie. Der Rezidivvergleich sportlich aktiver und nicht/wenig aktiver Jugendlicher zeigte eine Reluxationshäufigkeit von 82:30\% [36].

\section{Begleitverletzungen}

Burkhart u. De Beer [4] beobachteten eine Rezidivrate von $67 \%$ bei Patienten mit knöchernen Läsionen (Bankart-Fraktur, HillSachs-Läsion), bei Patienten ohne knöcherne Begleitverletzungen dagegen nur eine Reluxationsrate von $4 \%$. Sie sahen darin den Grund für das Versagen der arthroskopischen Stabilisierung beim Vorliegen knöcherner Verletzungen [4]. Eine Operationsindikation bei in 3-22\% aller traumatischen Schulterluxationen auftretender Bankart-Fraktur besteht ab einer Defektgröße von $25 \%$ der kraniokaudalen Glenoidlänge, da die fehlende Auflagefläche eine deutlich vermehrte Translation mit Rezidivinstabilität provoziert [22, 30, 37]. Hill-Sachs-Defekte sind $a b$ einer Größe von 1/3 der Humeruskopfzirkumferenz oder bei zentraler Lage reluxationsrelevant und daher therapiebedürftig [21, 25].

Bei Patienten höheren Alters werden im Zusammenhang mit einer traumatischen Schulterluxation in 10-30\% Abrissfrakturen oder Infraktionen des Tuberculum majus beobachtet [20, 27, 33]. Dieses

Trauma Berufskrankh 2006 -8[Suppl 3]:S258-S263

DOI 10.1007/s10039-005-1067-x

○) Springer Medizin Verlag 2005

C. Voigt $\cdot$ H. Lill

\section{Abgrenzungsprobleme bei Schulterluxationen}

\section{Zusammenfassung}

Im folgenden werden die verschiedenen Formen der Schulterluxationen und -instabilitäten mit typischen Begleitverletzungen durch die klinische und bildgebende Diagnostik differenziert und auf dem Hintergrund prognostisch relevanter Faktoren spezifische Therapien abgeleitet.

Die klinische Diagnostik ist Grundlage der Klassifizierung und einer pathologiekonformen Behandlung. Im klinischen Alltag hat sich die Einteilung nach Gerber bewährt. Die dynamischen Instabilitäten werden nach Richtung, Hyperlaxität und Willkürlichkeit differenziert. Posttraumatisch sind v. a. unidirektionale Instabilitäten ohne (B2) und mit Hyperlaxität (B3) zu beobachten. Sie werden bei <30-jährigen Patienten primär operativ, zunehmend arthroskopisch, stabilisiert. Bei >30-Jährigen ohne wesentliche Begleitverletzungen wird konservativ behandelt. Begleitende Rotatoren- manschettenrupturen sollten in der MRT verifiziert und frühzeitig rekonstruiert werden. Ossäre Begleitverletzungen wie BankartFrakturen und Hill-Sachs-Läsionen sind $a b$ einer Größe von 25\% der kraniokaudalen Glenoidlänge bzw. >1/3 der Humeruskopfzirkumferenz reluxationsrelevant und somit operationsbedürftig. Im Zusammenhang mit Schulterluxationen auftretende Tuberculum-majus-Frakturen gehen mit einer geringen Reluxationsrate einher, sodass eine Operationsindikation nur bei nach Reposition persistierender Dislokation besteht. Multidirektionale Instabilitäten mit Hyperlaxität (B5) werden mit überwiegend guten Ergebnissen konservativ behandelt.

\section{Schlüsselwörter}

Schulterluxation · Begleitverletzungen . Reluxationsrate · Prognosefaktoren . Therapieverfahren

\section{Problems of differential diagnosis in shoulder dislocation}

\section{Abstract}

Shoulder dislocations and instabilities and the typical concomitant injuries seen on clinical and radiological examination are presented in this paper, and specific therapeutic procedures are elaborated. Correct classification and therapy are based on the clinical examination and treatment in keeping with the pathology observed. The Gerber classification system has proved its worth in day-to-day clinical life. Dynamic instabilities are differentiated by direction, the presence or absence of hyperlaxity, and arbitrariness. Posttraumatic instability is mostly unidirectional, without (B2) or with (B3) hyperlaxity. In patients $<30$ years of age the primary therapy is surgical, and arthroscopic techniques are being applied with increasing frequency. Patients $>30$ years of age who have no serious concomitant injuries can be treated conservatively. When associated rotator cuff tears - which are often seen in patients
$>40$ years of age - are suspected MRI should be performed to confirm this diagnosis, and early reconstruction must be carried out in the case of such lesions. Bony lesions such as Bankart fracture $(>25 \%$ of the craniocaudal distance) or Hill-Sachs lesion (1/3 of the humeral head circumference) predispose to redislocation and therefore need surgical management. For shoulder dislocations associated with fracture of the greater tuberosity the recurrence rate is lower, and in these cases surgical treatment should not be performed except when dislocation persists after reduction. Multidirectional instabilities with hyperlaxity (B5) can be treated by conservative techniques with predominantly good results.

\section{Keywords}

Shoulder dislocation - Concomitant injuries . Recurrence rate - Prognostic factors .

Treatment 
Klientel zeigt vergleichsweise niedrigere Reluxationsraten [27, 38].

Begleitende Rotatorenmanschettenrupturen betreffen ebenfalls vorwiegend ältere Patienten. Ribbans et al. [31] sahen bei $63 \%$ der $>50$-Jährigen eine Rotatorenmanschettenläsion nach Erstluxation. Sie erhöht das Rezidivrisiko [32]. Die tatsächliche Inzidenz von Rotatorenmanschettenrupturen post luxationem ist schwer zu erfassen, da mit zunehmendem Alter häufig auch asymptomatische Rotatorenmanschettendefekte zu beobachten sind [35]. Daher ist nach Schultererstluxation bei $\mathrm{Pa}$ tienten $>40$ Jahren eine dahingehende initiale Diagnostik (MRT) obligat [26, 27].

Auch begleitende Nervenverletzungen (N. axillaris $>N$. suprascapularis $>N$. radialis $>N$. musculocutaneus) finden sich, häufig in Verbindung mit ausgeprägten Hämatomen, vornehmlich beim älteren Patienten [6]. Die Häufigkeitsangaben schwanken zwischen 8 und 45\% nach elektrophysiologischen Messungen [6, 27]. Die meisten sind innerhalb von 4 Monaten reversibel [6].

Liegt die Trias von traumatischer Schulterluxation, Rotatorenmanschettenruptur und N.-axillaris-Schädigung vor, spricht man von der "terrible triad of the shoulder" [16].

\section{Differenzialtherapie}

Das therapeutische Vorgehen richtet sich nach dem vorliegenden Klassifikationsstadium nach Gerber-Nyffeler [13] (• Tabelle 1) und den beschriebenen Prognosefaktoren.

\section{Operation}

Eine Notfallindikation stellen begleitende Gefäßschäden und verhakte Luxationen dar. Absolute Operationsindikation besteht bei nicht retinierbarer Luxation, Bankart-Fraktur (>25\% der kraniokaudalen Glenoidlänge), dislozierter Tuberculum-majus-Fraktur und M.-subscapularisRuptur [17, 25].

Die primäre operative Stabilisierung der traumatischen Erstluxationen ist bei Patienten <25 Jahre, einem hohen sportlichen Anspruch, einem adäquaten Trauma mit Fremdreposition, bei Hill-Sachs-Defekt (>1/3 der Humeruskopfzirkumferenz, zentraler Lage) und der sehr seltenen Luxatio erecta (untere Luxation durch Hyperabduktion) angezeigt $[17,25,27]$. Rotatorenmanschettendefekte sollten frühzeitig rekonstruiert werden $[14,17,25,27]$.

Kommt es nach konservativer Therapie zu Rezidiven, persistieren Subluxation, subjektiver Instabilität oder pathologischen Stabilitätstests, ist die sekundäre operative Stabilisierung anzustreben.

Die operative Stabilisierung umfasst eine Rekonstruktion und Refixation des Labrum-Kapsel-Komplexes mit Kapselshift. Dieser kann in klassischer Weise offen als Bankart-Operation oder arthroskopisch durchgeführt werden. Die Metaanalyse von Mothadi et al. [29] favorisiert unter Auswertung entsprechender Publikationen von 1966-2003 das offene Vorgehen mit geringeren Reluxationsraten gegenüber arthroskopischen Verfahren (transglenoidal, „suture tacs“) bei posttraumatisch rezidivierender anteriorer Instabilität. Aktuell werden unter dem Einsatz von Nahtankern vergleichbare Reluxationsraten nach arthroskopischer Stabilisierung mit geringerer Morbidität und verbesserter postoperativer Beweglichkeit beschrieben [7, 24], sodass zunehmend die arthroskopische Stabilisierung empfohlen wird (- Abb. 3). Grenzen des arthroskopischen Verfahrens sind eine unzureichende Qualität des Kapselgewebes (?), große BankartFrakturen, einhakende Hill-Sachs- sowie HAGL-Läsionen [23].

\section{Konservative Behandlung}

Eine konservative Behandlung ist nach traumatischer Schulterluxation bei Patienten $>30$ Jahre ohne relevante knöcherne Begleitverletzungen und $>40$-jährigen Patienten ohne begleitende Rotatorenmanschettenrupturen indiziert. Der Einfluss der Immobilisationsdauer auf die $\mathrm{Re}$ zidivrate wird diskutiert. Eine längere Ruhigstellung scheint nicht sinnvoll zu sein. Es sollte eine frühzeitige kontrollierte Physiotherapie mit initialen isometrischen Übungen erfolgen [27].

\section{Besonderheiten bei multidirektio- naler Instabilität (MDI)}

Die MDI ohne Hyperlaxität ist sehr selten. Für die MDI mit Hyperlaxität liefert die konservative Therapie in bis zu 80\% ,sehr gute“ und "gute“ Ergebnisse $[2,5]$. Ein operatives Vorgehen ist nur bei nach mindestens 6-monatiger kontrollierter Physiotherapie persistierender Instabilität indiziert. Der arthroskopische posterior-anteriorinferiore Kapselshift mit Verschluss des Rotatorenintervalls wird empfohlen [10, 19]. Eigene Untersuchungen zeigen überwiegend „sehr gute“ 1-Jahres-Ergebnisse nach diesem Verfahren bei jungen Überkopfsportlern.

\section{Fazit für die Praxis}

Die Gerber-Klassifikation eignet sich für die Einteilung dynamischer Schulterinstabilitäten, da sich anhand der Instabilitätsform eine geeignete Therapie ableiten lässt. Chronisch verhakte Luxationen (B1), unidirektionale Instabilitäten ohne und mit Hyperlaxität (B2, B3) sowie die sehr seltenen multidirektionalen Instabilitäten ohne Hyperlaxität (B4) sind die Domäne der operativen Therapie. Neben dem offenen Vorgehen bei B1-Instabilitäten werden für B2- bis B4-Instabilitäten zunehmend die arthroskopische Rekonstruktion und Refixation des Kapsel-Labrum-Komplexes mit Kapselshift empfohlen. Multidirektionale Instabilitäten mit Hyperlaxität werden vornehmlich konservativ behandelt. Operative Maßnahmen (arthroskopischer posterior-anterior-inferiorer Kapselshift und Intervallverschluss) erfolgen nur nach erfolgloser kontrollierter Physiotherapie. Willkürliche symptomatische Instabilitäten werden konservativ behandelt.

Patientenalter, Aktivitätsgrad und Begleitverletzungen beeinflussen die Prognose und somit das therapeutische Vorgehen nach traumatischer Schulterluxation. Bei Erstluxation <30-jähriger sowie sportlich aktiven Patienten ist eine primäre operative Stabilisierung indiziert; >30-Jährige ohne wesentliche Begleitverletzungen können, da sie deutlich niedrigere Reluxationsraten aufweisen, konservativ behandelt werden. Begleitende Rotatorenmanschettenrupturen, vornehmlich bei $>40$ jährigen Patienten, sollten frühzeitig rekonstruiert werden. Bankart-Frakturen und Hill-Sachs-Läsionen sind ab einer GröBe von 25\% der kraniokaudalen Glenoidlänge bzw. > 1/3 der Humeruskopfzirkum- 
ferenz operationsbedürftig. Im Zusammenhang mit Tuberculum-majus-Frakturen wird ein deutlich verringertes Reluxationsrisiko beobachtet, sodass diese nur bei Dislokation osteosynthetisch zu versorgen sind.

\section{Korrespondierender Autor Dr. C. Voigt}

Klinik für Unfall- und Wiederherstellungschirurgie, Friederikenstift, Humboldtstraße 5, 30169 Hannover E-Mail: christine.voigt@friederikenstift.de

Interessenkonflikt: Es besteht kein Interessenkonflikt. Der korrespondierende Autor versichert, dass keine Verbindungen mit einer Firma, deren Produkt in dem Artikel genannt ist, oder einer Firma, die ein Konkurrenzprodukt vertreibt, bestehen. Die Präsentation des Themas ist unabhängig und die Darstellung der Inhalte produktneutral.

\section{Literatur}

1. Altcheck DW, Warren RF, Skyhar MJ et al. (1991) Tplasty modification of the Bankart procedure for multidirectional instability of the anterior and inferior types. J Bone Joint Surg Am 73-A: 105-112

2. An YH, Friedman RJ (2000) Multidirectional instability of the glenohumeral joint. Orthop Clin N Am 31: $275-285$

3. Brunner U (2002) Klinische Untersuchung der Schulter. In: Habermeyer P (Hrsg) Schulterchirurgie, 3. Aufl. Urban \& Fischer, München, S 45-69

4. Burkhart SS, De Beer JF (2000) Traumatic glenohumeral bone defects and their relationship to failure of arthroscopic Bankart repairs: significance of the inverted-pear glenoid and the humeral engaging Hill-Sachs lesion. Arthroscopy 16: 677-694

5. Burkhead WZ Jr, Rockwood CA Jr (1992) Treatment of instability of the shoulder with an exercise program. J Bone Joint Surg Am 74-A: 890-896

6. De Laat EA, Visser CP, Coene LN et al. (1994) Nerve lesions in primary shoulder dislocations and humeral neck fractures. A prospective clinical and EMG study. J Bone Joint Surg Br 76-B: 381-383

7. Fabbriciani C, Milano G, Demontis A et al. (2004) Arthroscopic versus open treatment of Bankart lesion of the shoulder: a prospective randomized study. Arthroscopy 20: 456-462

8. Fuchs B, Jost B, Gerber C (2000) Posterior-inferior capsular shift for the treatment of recurrent, voluntary posterior subluxation of the shoulder. J Bone Joint Surg Am 82-A: 16-25

9. Gagey OJ, Gagey N (2001) The hyperabduction test. J Bone Joint Surg Br 83-B: 69-74

10. Gartsman GM, Roddey TS, Hammerman SM (2001) Arthroscopic treatment of multidirectional glenohumeral instability: 2- to 5-year follow up. Arthroscopy 17: 236-243

11. Gerber C (1988) Les instabilites de l'e'paule. Cahiers dénseignement de la SOFCOT. Expansion Scientifique Francaise Paris 33: 51-74

12. Gerber C (1997) Observations on the classification of instability. In: Warner JP, lannotti JP, Gerber C (eds) Complex and recision problems in shoulder surgery. Lippincott-Raven, Philadelphia, pp 9-19
13. Gerber C, Nyffeler RW (2002) Classification of glenohumeral joint instability. Clin Orthop 400: 65-76

14. Glombik TM (2004) Instabile Schulter. Klassifikation und Therapie - unter besonderer Berücksichtigung der traumatischen vorderen Schultererstluxation. Trauma Berufskrankh 6: 126-133

15. Gumina S, Postacchini F (1997) Anterior dislocation of the shoulder in elderly patients. J Bone Joint Surg Br 79-B: 540-543

16. Guven O, Akbar Z, Yalcin S et al. (1994) Concomitant rotator cuff tear and brachial plexus injury in association with anterior shoulder dislocation: unhappy triad of the shoulders. J Orthop Trauma 8: 429-430

17. Habermeyer P, Jung D, Ebert T (1998) Behandlungsstrategie bei der traumatischen vorderen Erstluxation der Schulter. Unfallchirurg 101: 328-341

18. Hawkins RJ, Bokor DJ (1990) Clinical evaluation of shoulder problems. In: Rockwood CA, Matsen FA III (eds) The shoulder, vol 1. Saunders, Philadelphia, pp 149-177

19. Hewitt M, Getelman MH, Snyder SJ (2003) Arthroscopic management of multidirectional instability: pancapsular placation. Orthop Clin N Am 34: 549557

20. Hovelius L, Eriksson K, Fredin H et al. (1983) Recurrences after initial dislocation of the shoulder. Results of a prospective study of treatment. J Bone Joint Surg Am 65-A: 343-349

21. Hovelius L, Augustini BG, Fredin H et al. (1996) Primary anterior dislocation of the shoulder in young patients. J Bone Joint Surg Am 78-A: 1677-1684

22. Itoi E, Lee SB, Berglund LJ et al. (2000) The effect of a glenoid defect on antero-inferiore stability of the shoulder after Bankart repair: a cadaveric study. J Bone Joint Surg Am 82-A: 35-46

23. Jäger A, Braune C, Eisenhart-Rothe RMO von et al. (2004) Arthroskopische vs. offene Stabilisierung der Schulter. Arthroskopie 17: 171-178

24. Kim SH, Ha Ki (2002) Bankart repair in traumatic anterior shoulder instability: open versus arthroscopic technique. Arthroscopy 18: 755-763

25. Lichtenberg S, Magosch $P$, Habermeyer $P$ (2005) Traumatische vordere Schulterluxation. Unfallchirurg 108: 299-314

26. Lill H, Lange H, Reinbold WD et al. (1997) MRT-Arthrographie - Verbesserte Diagnostik bei Schultergelenkinstabilitäten. Unfallchirurg 100: 186-192

27. Lill H, Verheyden P, Korner J et al. (1998) Konservative Behandlung nach traumatischer Schultererstluxation? Chirurg 69: 1230-1237

28. Matsen FA, Thomas SC, Rockwood CA (1990) Glenohumeral Instability. In: Rockwood CA, Matsen FA (eds) The shoulder, vol 1. Saunders, Philadelphia, pp 526-622

29. Mothadi NGH, Bitar IJ, Sasyniuk TM et al. (2005) Arthroscopic versus open repair for traumatic anterior shoulder instability: a meta-analysis. Arthroscopy 21: 652-658

30. Norlin R (1993) Intraarticular pathology in acute, first-time anterior shoulder dislocation: an arthroscopic study. Arthroscopy 9: 546-549

31. Ribbans WJ, Mitchell R, Taylor GJ (1990) Computerised arthrotomography of primary anterior shoulder dislocation of the shoulder. J Bone Joint Surg Br 72-B: 181-186

32. Robinson CM, Kelly M, Wakefield AE (2002) Redislocation of the shoulder during the first week after a primary anterior dislocation: risk factors and results of treatment. J Bone Joint Surg Am 84-A: $1552-1559$

33. Rowe CR (1956) Prognosis in dislocations of the shoulder. J Bone Joint Surg Am 38-A: 957-977
34. Scheibel M, Imhoff A (2004) Definition, Klassifikation und klinische Diagnostik der instabilen Schulter. Arthroskopie 17: 139-145

35. Sher JS, Uribe JW, Posada A et al. (1995) Abnormal findings on magnetic resonance images of asymptomatic shoulders. J Bone Joint Surg Am 77-A: 10-15

36. Simonet WT, Cofield RH (1984) Prognosis in anterior shoulder dislocation. Am J Sports Med 12: 19 24

37. Taylor DC, Arciero RA (1997) Pathologic changes associated with shoulder dislocation. Am J Sports Med 25: 306-311

38. te Slaa RL, Wijffels MP, Brand R et al. (2004) The prognosis following acute primary glenohumeral dislocation. J Bone Joint Surg Br 86-B: 58-64

39. Wheeler JH, Ryan JB, Arciero RA et al. (1989) Arthroscopic versus non-operative treatment of acute shoulder dislocations in young athletes. Arthroscopy 5: 213-217 